\title{
Pratiques solidaires sous l'angle de la territorialité
}

\author{
Jean-Marc Fontan, Yanick Noiseux et Marco Silvestro \\ Université du Québec à Montréal
}

\section{Introduction}

$\mathcal{N}$

ous présentons ici les résultats préliminaires d'un programme de recherche portant sur l'étude de pratiques québécoises de production et d'échange solidaires. Les pratiques que nous avons étudiées permettent d'analyser des innovations sociales inscrites dans des activités économiques mettant en scène une dimension territoriale $^{1}$. La recherche a été menée entre l'automne 2003 et l'hiver 2005 par les membres du Collectif d'étude des pratiques solidaires (CEPS), lequel est affilié au Centre de recherche sur les innovations sociales (CRISES) ${ }^{2}$.

Nous avons retenu des pratiques solidaires québécoises récentes qui répondent à un certain nombre de critères innovateurs dans la façon de penser le rapport salarial, le rapport à la démocratie, le rapport de genre, le rapport à l'environnement et le rapport au territoire. Concrètement, une initiative qui proposerait de penser de façon novatrice la solidarité entre un producteur et un consommateur est plus propice à retenir notre attention qu'une pratique qui propose une approche « classique » de mise en rapport du producteur et du consommateur. Dans le même sens, nous portons une attention particulière aux initiatives définissant autre-

\section{Il nous est apparu que les valeurs à la base de la solidarité différaient en fonction du rapport à la territorialité.}

qu'avaient les membres du collectif de recherche. Toutefois, avant d'inclure une expérience dans notre échantillon, nous nous sommes assurés qu'elle était relativement récente (horizon d'une vingtaine d'années) et qu'elle portait une intention d'alternativité par rapport au système économique dominant. En raison du caractère exploratoire de la recherche, nous avons choisi d'être, de prime abord, plus inclusifs qu'exclusifs, les véritables critères de l'alternativité d'une pratique ne pouvant être définis ex ante.

Cet article est divisé en deux grandes sections. La première présente les données concernant le lien au territoire des pratiques solidaires étudiées. La deuxième met en relation les innovations sociales relevées et la question du développement des territoires. En conclusion, nous évaluons l'ensemble de cette démarche exploratoire en en faisant ressortir les limites et les potentialités.

\section{La manifestation spatiale des solidarités}

Dans cette première section, nous utilisons la dimension spatiale pour situer la portée des pratiques solidaires étudiées. Il nous est apparu que les valeurs à la base de la solidarité différaient en fonction du rapport à la territorialité. Nous avons donc classé les différentes initiatives à partir de ment des notions telles que la valeur, le marché, la division du travail, la propriété (ses droits et ses nondroits), la démocratie organisationnelle, le rapport à l'environnement, etc. Enfin, nous étudions des expériences qui pensent différemment la solidarité à initier entre des territoires de proximité (ville et campagne) ou des territoires éloignés (le Nord et le Sud).

Le choix des vingt initiatives a été réalisé à partir d'un échantillon dit de convenance, soit en fonction des connaissances et des expériences de terrain la portée spatiale de la solidarité exprimée en leur sein (figure 1).

En observant la distance géographique qui sépare l'organisme (ou l'individu) qui effectue la pratique de celui qui en bénéficie, nous sommes en mesure de cerner la portée spatiale de la solidarité pour chacune des vingt initiatives. Notre critère de mesure correspond à des appellations géographiques générales. Ainsi, nous identifions trois dimensions spatiales pour caractériser des rapports solidaires: les dimensions 
Nord-Nord, Nord-Sud et celle dite "globale ». La dimension Nord-Nord, celle que nous avons davantage explorée, a été subdivisée afin de considérer ses composantes locale, régionale, provinciale, nationale et internationale.

En analysant les initiatives sélectionnées, nous avons repéré des constantes qui sont spécifiques aux configurations spatiales de la solidarité. Nous avons avancé une hypothèse à ce sujet : lorsque la portée spatiale d'une pratique solidaire varie, il en est de même des valeurs solidaires et de la règle d'échange qui y sont associées.

Les raisons pour expliquer cela sont multiples. Par exemple, nous croyons que la différence de niveau de vie et le degré de conscience sociale des acteurs impliqués peuvent influencer cette variation. Une autre observation tient au fait que les moyens déployés par les acteurs varient également selon la portée spatiale de leur pratique solidaire.

Figure 1

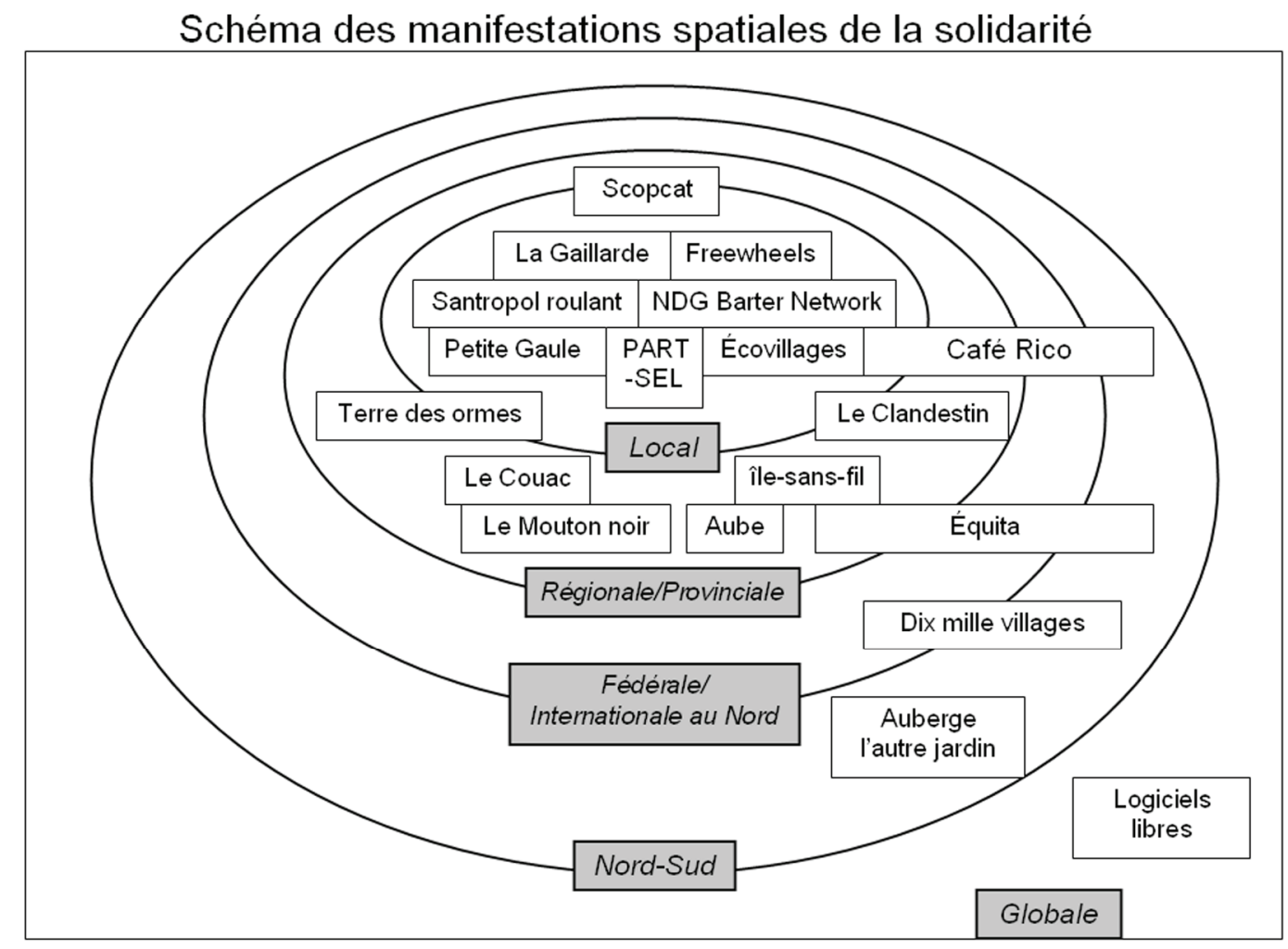

\section{Rapport Nord-Nord}

Plusieurs initiatives opèrent sur une base solidaire locale. Ce mode signifie que la pratique solidaire fonctionne surtout dans un contexte de proximité géographique entre les acteurs, où les rapports sociaux se vivent de façon plus directe et communautaire. Il est courant de voir dans cette dimension une dynamique de réciprocité où la pratique solidaire profite à l'ensemble des acteurs impliqués. La solidarité basée sur la réciprocité, qui ressemble sur plusieurs points au troc, prend alors une forme moins altruiste qu'une solidarité internationale davantage unidirectionnelle. Par exemple, dans les systèmes d'échanges locaux
(SEL), chacune des parties impliquées profite d'une reformulation des termes de l'échange qui cherche entre autres à combler le fossé entre les classes sociales quant à la rémunération en fonction des compétences et de l'éducation. Ainsi, une heure de gardiennage équivaut à une heure de services comptables.

La solidarité régionale s'observe au travers de pratiques concernant une région en particulier ou quelques régions limitrophes. Cette dimension ne fait plus autant appel à une solidarité communautaire, mais plutôt à un sentiment d'appartenance territoriale plus large (grand centre urbain, région de la province) qui relève de l'historicité québécoise. Au sein de la dimension 
régionale, nous avons identifié une autre configuration spatiale, celle de la solidarité entre les milieux rural et urbain. L'exemple type est sans doute l'initiative Terre des Ormes où des paysans qui pratiquent l'agriculture soutenue par la communauté (ASC) ${ }^{3}$ invitent les partenaires citadins à travailler sur leur terre en échange d'une expérience de vie en milieu rural. La solidarité liée à cette pratique, à la fois locale et régionale, se base sur une philosophie qui cherche à combler le fossé culturel entre les grands centres urbains et les régions rurales.

Dans les pratiques Nord-Nord identifiées, la dimension nationale et internationale des manifestations spatiales de la solidarité apparaît au travers des réseaux liés au commerce équitable, notamment quant à la certification des coopératives de producteurs du Sud. Nous avons aussi inséré les réseaux solidaires formés de commerçants de produits équitables s'organisant parfois sur cet horizon pour l'achat du café équitable et la gestion de leurs boutiques.

\section{Rapport Nord-Sud}

La chaîne du commerce équitable répond aussi d'une dynamique Nord-Sud. Les trois initiatives identifiées sont des promoteurs, distributeurs et importateurs 10000 villages, Café Rico, Équita. Une quatrième initiative, L'Auberge l'Autre jardin, a pour mission de participer au financement de l'organisme Carrefour Tiers-Monde, de Québec, et offre par ailleurs des produits équitables dans sa boutique. La solidarité en jeu dans ces initiatives de commerce équitable se base sur une critique, plus ou moins acerbe dépendamment des cas étudiés, du système du commerce international qui est perçu comme générateur d'injustices socioéconomiques. Selon le discours porté par ces initiatives, les pratiques solidaires Nord-Sud rapportent surtout aux communautés des pays du Sud.

\section{Nous observons que les organismes dont la solidarité s'exerce du Nord au Sud réalisent également plusieurs actions solidaires au Nord.}

\section{Rapport global}

Les logiciels libres, dont les exemples les plus répandus sont sans doute le système d'exploitation Linux et le fureteur Mozilla-Firefox, constituent un cas atypi- que dans notre recherche. La solidarité qui y est invoquée se situe au niveau global. Leur caractère immatériel fait en sorte qu'ils peuvent, considérant le développement actuel des moyens de communication, traverser les frontières des États sans trop rencontrer d'obstacles.

La solidarité exprimée au sein de ces pratiques se situe dans la volonté de partager les résultats du travail accompli. Cette solidarité est à deux volets: d'une part, la possibilité de distribuer librement (gratuitement ou non) et, d'autre part, la possibilité que le produit soit édité par quiconque en possède les moyens. La «commercialisation » reste possible, mais on ne peut pour autant revendiquer la propriété du code utilisé (qui doit rester disponible).

Les logiciels libres représentent, en quelque sorte, une forme de dépassement du principe de la propriété privée et des droits afférents à l'origine d'un produit ou d'une invention (brevets et patentes). Même si le « mouvement» du libre est équivoque, la philosophie qui en est à l'origine est basée sur l'idée de partage universel du savoir. Cependant, ce partage est encore inégal et apparaît dans la "fracture numérique », un clivage flagrant à l'échelon mondial malgré les avancées importantes dans les pays du Sud.

Deux observations pour conclure cette partie. Tout d'abord, nous observons que les organismes dont la solidarité s'exerce du Nord au Sud réalisent également plusieurs actions solidaires au Nord. Par exemple, l'organisme de commerce équitable Café Rico mise sur la consolidation des ressources locales. Ainsi, la boutique de Montréal sert de point de chute à l'agriculture soutenue par la communauté et vend certains produits biologiques ou artisanaux québécois. Cela pourrait s'expliquer par le fait que certains acteurs voient un avantage à fortifier leur ancrage local pour ensuite mieux projeter leur action solidaire dans les pays du Sud.

Ensuite, il est intéressant de remarquer que la solidarité repose souvent sur une motivation plus ou moins abstraite selon qu'elle se situe à l'échelle locale ou à l'échelle Nord-Sud. La solidarité Nord-Sud repose davantage sur des principes abstraits qui sont porteurs d'une action plus globale visant à réduire les inégalités dans plusieurs régions de la planète, tandis que les projets locaux misent davantage sur une solidarité à caractère communautaire où toutes les parties im- 
pliquées bénéficient de l'échange. Par ailleurs, les pratiques solidaires Nord-Sud tendent à prendre la forme d'actions charitables, alors qu'au niveau local ces pratiques correspondent davantage à une forme d'entraide.

\section{À partir du moment où le fait de consommer du café commence à être perçu, tant au Nord qu'au Sud, comme une relation sociale entre un producteur et un consommateur, la question de la territorialité se pose.}

\section{Constats réflexifs sur les innovations mises en scène par rapport au territoire}

Nous dégageons plusieurs éléments d'analyse des études de cas réalisées.

Premièrement, nombre de pratiques font du territoire une "clé sociale» pour comprendre les modalités d'échange qu'elles mettent en scène. À titre indicatif, une pratique telle l'ASC, avec le projet Terre des Ormes, ou encore une pratique telle la fabrication d'un fromage artisanal, Le Clandestin, visent essentiellement à repenser les liens entre la ville et la campagne. Ces liens sont avant tout perçus 1) dans la qualité du produit à consommer, 2) dans la profondeur de la relation entre le producteur et le consommateur et 3) dans la qualité du réseau qui soutient l'échange. Il s'agit de ne pas penser le produit et l'échange dans les termes d'une relation conventionnelle de marché, soit en fonction des retombées économiques, de la réduction des coûts, de la recherche d'économie d'échelle et de la production d'externalités. Au contraire, une médiation est invoquée entre le producteur et le consommateur sur ces questions. Ces dernières ne sont pas banalisées et sont prises en compte dans l'échange : ce qui force, par exemple, l'expression démocratique et la prise de parole entre le producteur et le consommateur et non une médiation par des « experts $\gg$.

De plus, l'économie marchande libérale pose une déconnection entre le producteur et le consommateur. Le fait que cette économie ne se préoccupe pas d'informer le consommateur sur la provenance des produits est révélateur. Savoir que des pastèques sont produites dans le désert est une information importante permettant au consommateur d'être conscient qu'il opte pour un produit exigeant des coûts inhabituels et une externalisation d'effets négatifs pour l'environnement. Savoir que le bien ou le service provient d'une zone franche où les conditions de travail rencontrées sont indécentes est aussi important. Ne pas prendre en considération cet aspect informationnel contribue à rendre invisible la variable territoriale dans l'échange marchand.

Deuxièmement, la prise en considération du territoire, de l'importance de la proximité dans la relation d'échange permet d'observer un besoin de redéfinition des modalités de régulation dans les processus de production ou de consommation. À titre indicatif, le commerce international du café est fondé sur la nonprise en compte de la proximité entre le producteur du Sud et le consommateur du Nord. L'intermédiaire, une multinationale du café, se donne le droit d'agir au nom du «bien-être de tous »: de l'emploi dans les plantations au Sud et du café dans les tasses au Nord. À partir du moment où le fait de consommer du café commence à être perçu, tant au Nord qu'au Sud, comme une relation sociale entre un producteur et un consommateur, la question de la territorialité se pose : les acteurs pensent appartenir à un espace commun et se doivent de co-définir leurs relations dans cet espace. Il en découle de nouveaux termes pour réguler les échanges dans une perspective d'ajustement du déséquilibre qu'engendrait le mode antérieur de production et de commercialisation du café. Toutefois, notre travail montre bien que le processus de prise en compte de la proximité dans une perspective d'ajustement des termes de l'échange demeure incomplet. D'autres innovations doivent suivre pour en arriver à un ajustement plus équitable et écologique.

Troisièmement, les innovations technologiques, économiques, organisationnelles et sociales mises en scène dans les expériences étudiées permettent un apprentissage et une solidarité bidirectionnelle : du centre vers la périphérie et de la périphérie vers le centre. $\mathrm{Au}$ clivage solidaire et cognitif qu'impliquait les phases coloniale puis fordiste de mise en relation des populations du monde, se juxtaposent des passerelles qui rendent possible un apprentissage au Nord à partir des avancées et des innovations pensées et appliquées par des acteurs sociaux du Sud. L'inverse est aussi vrai. Il en est de même quant aux solidarités. Par l'ASC, des agriculteurs (généralement localisés dans une périphérie ou une péri-urbanité) accueillent une solidarité de consommateurs (généralement localisés 
dans un centre) qui jugent plus appropriés de courtcircuiter les chaînes de distribution par un geste d'investissement représenté par l'achat en avance d'une partie de la récolte du producteur. La solidarité précède ainsi l'acte de consommation.

Quatrièmement, une des particularités de nombre de pratiques étudiées permet de mettre en évidence la façon dont des pratiques solidaires sont pensées en fonction des forces et des faiblesses de leur milieu. Plusieurs initiatives cherchent à recréer une solidarité de proximité : les cas de Freewheels, du défunt café La Petite Gaule (ou de l'Utopik) ou encore de Santropol Roulant. Dans ces cas, il y a certes des activités économiques, donc des échanges à caractère économique, mais ils sont définis et élaborés comme le moyen de parvenir à une fin autre. L'économique est subordonné au social.

Cinquièmement, si l'économique est subordonné au social, il n'empêche que les pratiques montrent bien l'importance de l'économique pour résoudre ou s'attaquer à des questions sociales. L'innovation repose alors sur l'ouverture à la mixité. En témoignent des expériences comme L'Auberge l'Autre Jardin où l'activité hôtelière est exercée pour financer les activités courantes d'une organisation de coopération internationale: Carrefour Tiers-Monde. Cette mixité se construit dans la combinaison d'objectifs sociaux, économiques, politiques et culturels et démontre qu'il est non seulement possible mais aussi légitime de ne pas externaliser les dimensions sociale, politique et culturelle lorsqu'on s'investit dans un projet à caractère ou à dominante économique. Elle se construit aussi dans la mixité des espaces : l'auberge est sur le territoire de la ville de Québec, elle représente une activité locale qui se fait au profit d'une organisation qui travaille à l'échelon des relations internationales. Si la tendance à la monétarisation et à la création de richesse est bien présente dans les expériences étudiées, nous relevons aussi une autre tendance: celle d'ouvrir la question du droit de gérance sur les activités de production. La mouvance du libre ou encore les pratiques de troc témoignent d'une façon de produire un bien ou un service sans recourir à la médiation monétaire, sans se référer à la valeur d'échange conférée par la diplomation ou le statut social ou encore à la médiation du droit de propriété.

Sixièmement, la question écologique apparaît en filigrane et toutes les pratiques étudiées y réfèrent d'une façon ou d'une autre. Ce point est important dans le contexte actuel puisque la dégradation causée par les activités humaines sur nombre d'écosystèmes a atteint un niveau tel que ce problème est devenu, avec la pauvreté, une des grandes questions sociales caractérisant notre époque et auxquelles il importe d'apporter des solutions satisfaisantes. Sur la question environnementale, le rapport au local est central. La solution consistant à externaliser les coûts que nous ne voulons pas introduire dans l'activité productive (les résidus polluants par exemple) ou dans l'activité consommative (les déchets de surconsommation, le transport) trouve facilement un lieu de réponse à l'échelle de l'entreprise localisée ou de l'unité de consommation que représente le ménage. Le local, comme espace relationnel supportant un milieu de vie, est aussi un lieu de régulation et de mise en scène d'innovations culturelles, en termes de grandes orientations ou de charte morale, à partir desquelles nous pouvons contraindre toute unité de production ou de consommation à limiter à sa plus simple expression l'externalisation des « coûts environnementaux ».

\section{En guise de conclusion : vers d'autres perspectives de recherche ${ }^{4}$}

L'étude de vingt pratiques solidaires québécoises nous a permis d'analyser de nouvelles façons de penser et d'actualiser des rapports sociaux à vocation économique. L'objectif de cette première étape de notre projet de recherche était d'identifier des expériences types ayant un potentiel d'alternativité, susceptibles de proposer des éléments de reconfiguration du système économique dominant ou de présenter de nouveaux éléments de grammaire propices à la construction de nouveaux modèles de développement. Les résultats nous permettent de valider notre intuition. Nous observons effectivement des éléments porteurs tant d'un potentiel d'alternativité que d'un potentiel de transformation du mode actuel de régulation au sein de la société québécoise.

Cette étude nous a aussi permis de valider la pertinence et la richesse de la démarche de travail que nous avons utilisée. Plusieurs éléments d'enseignement s'en dégagent. D'une part, l'approche par analyse territoriale nous informe sur les contraintes inhérentes liées à la portée spatiale d'une initiative. A contrario de ce que nous pensions, il persiste des éléments structuraux qui viennent contraindre les acteurs du Sud et du Nord dans leur volonté d'agir, dans la pro- 
duction de leur vivre ensemble, et qui les oblige parfois à redéfinir leur projet originel en regard de leur expérience de la contrainte. D'autre part, cette recherche comporte des limites évidentes puisque l'exploration effectuée a permis de dégager des connaissances sans nécessairement offrir assez d'informations pour déployer une analyse critique approfondie des données à notre disposition. Le fait de travailler uniquement à partir de témoignages d'acteurs nous rendait dépendants de leur discours. Le caractère exploratoire de notre démarche nous permettait de travailler en surface sans être en mesure de compléter le peu d'informations à notre disposition. D'où l'importance de procéder à partir d'entrevues en profondeur et de réaliser un travail minimal d'observation participante.

Bref, au sein des expériences étudiées, peut-on déceler la présence d'innovations propices à une redéfinition du mode de régulation actuel ? Notre réponse est oui. Nous observons la présence d'un mouvement de revendication remettant en cause le droit de propriété. Dans la veine des travaux de Pierre-Joseph Proudhon, il ne s'agit pas d'une revendication théorique, mais bien d'une expérimentation concrète d'un agir collectif construit à partir de l'idée générale du « libre » et de l'autogéré.

Dans cette voie, les expériences des SEL, mais aussi des logiciels libres, nous permettent de déceler la présence d'un mouvement de revendication autour de l'échange non monétaire, de mise à jour des modalités du troc, sur des bases collectives et non individuelles. Si l'idée de contourner l'utilisation de la monnaie comme étalon d'échange est intéressante, l'est aussi celle d'équité dans la façon de mesurer ce qui est échangé. Sur ce point, les initiatives étudiées laissent entendre qu'il peut y avoir ou non innovation, en fonction des compromis et conventions qui sont élaborés.

A contrario, nous avons aussi observé les limites d'expériences très en vogue de nos jours, celles liées au commerce équitable. Ces dernières proposent une nouvelle façon de faire le commerce tout en acceptant la légitimité du cadre régulatif du commerce international, lequel est fondé sur le principe de l'inéquitabilité des échanges économiques pour produire une plus-value financière. Par contre, tant le commerce équitable que les SEL et certaines pratiques de l'ASC témoignent des développements actuels au sein, par exemple, des rapports sociaux entre les consomma- teurs et les producteurs. Cet univers relationnel bien cloisonné sous le fordisme, avec des rôles étanches et bien définis, laisse maintenant place à une hybridation des rôles et à une redéfinition des frontières (consomm'acteur, pro'sommateur, etc.). Cette question reste à creuser.

\section{Nous observons la présence d'un mouvement de revendication remettant en cause le droit de propriété.}

Nous avons aussi observé la présence de différences au sein des logiques solidaires existantes au Sud et au Nord, et une difficulté de convergence ou de prise en compte respective de ces logiques. Cette observation nous invite à creuser davantage les dimensions culturelle, structurelle et spatiale de la solidarité. Cette piste n'était pas apparente au début de la recherche et devra être prise en considération dans les étapes ultérieures.

Enfin, le cas des écovillages nous montre comment persistent à travers le temps les expérimentations se réclamant du mouvement historique des utopies concrètes. L'étude des écovillages liée à celle de l'ASC offre une piste intéressante pour l'étude de la remise en question du système actuel d'occupation du territoire. Il est clair que ces expériences innovent en proposant des rapports sociaux édifiés non pas dans une logique de marché, mais de niches. Sur ce point, nous n'avons pas été en mesure de voir comment l'édification de niches peut constituer ou non une brèche pour le remodelage du mode actuel de régulation de l'économie.

Il serait difficile de conclure sans mentionner deux grands absents : l'État, comme appareil de régulation des sociétés, et la gouvernance, au sens foucaldien, comme une modalité d'action sur les actions. Les discours des expériences étudiées tiennent peu ou pas compte de l'État, comme s'il n'existait pas. Qu'en estil vraiment ? Quelle place 1'État prend-il aux yeux des acteurs concernés ? Nous n'avons que peu d'indications à ce propos et il nous faudra en tenir compte dans la poursuite de nos travaux.

De la même façon, les expériences étudiées parlent peu des rapports de pouvoir en leur sein, comme si le pouvoir était équitablement distribué ou approprié par 
les participants. Qu'en est-il vraiment ? Comment se prennent les décisions ? Comment se gèrent la différence, la dissidence, le désaccord et le conflit? Encore une fois, nous n'en savons guère à ce sujet.

Enfin, notre enquête ne nous a pas permis de mettre en lumière les mécanismes de formation, d'apprentissage et de socialisation mis en œuvre pour pérenniser et faciliter la reproduction des modalités de solidarité pratiquées par les acteurs.

La prise en considération de ces absences nous permettra de revoir nos outils méthodologiques, d'approfondir nos connaissances sur les subversivités et les alternativités mises en œuvre par des expériences solidaires. Nous pourrons ainsi mieux comprendre les mécanismes d'apprentissage et de socialisation, les façons de concevoir les rapports au marché et à l'avoir ainsi que les rapports à l'État et au pouvoir.

\section{Notes et références}

1 Le tableau 1 résume les caractéristiques des vingt initiatives étudiées.
2 Voir le rapport de recherche pour les résultats préliminaires complets : Leblanc, J., Y. Noiseux, M. Silvestro et al. (2005). «Pratiques solidaires dans la relation d'échange. Monographies d'initiatives québécoises », Cahier $d u$ CRI$S E S, \mathrm{n}^{\circ}$ ES0506, UQAM.

Disponible en ligne sur www.unites.uqam.ca/ceps/.

3 L'ASC consiste à mettre en lien un producteur agricole avec des consommateurs. Ceux-ci achètent d'avance la récolte de celui-là, l'aidant ainsi à financer ses activités.

4 Voici quelques références qui ont été utilisées pour le présent article et qui sont disponibles sur Internet :

Noiseux, Y. (2004). «Document de référence Internet dossier commerce équitable », Cahiers du CRISES, $\mathrm{n}^{\circ}$ ET0416, UQÀM. www.unites.uqam.ca/ceps/.

Proudhon, P.-J. (1840). Qu'est-ce que la propriété, Chicoutimi, coll. Les classiques des sciences sociales, UQAC. http://classiques.uqac.ca/classiques/Proudhon/la_propriete/ La_propriete.pdf

Silvestro, M. (2004). "Quelques synthèses et réflexions sur le commerce équitable ", Cahiers du CRISES, $\mathrm{n}^{\circ}$ ET0420, UQÀM. www.unites.uqam.ca/ceps/.

Silvestro, M. et J.-M. Fontan (2005). « Vivre demain dans nos luttes d'aujourd'hui! », Possibles, vol. 29, n 2, p. 100 à 117. www.unites.uqam.ca/ceps/.

\section{Tableau 1 - Présentation synthétique des vingt initiatives étudiées}

\begin{tabular}{|c|c|c|c|c|c|}
\hline $\begin{array}{l}\text { Champ } \\
\text { d'activité }\end{array}$ & $\begin{array}{l}\text { Secteur } \\
\text { d'activité }\end{array}$ & $\begin{array}{l}\text { Initiative } \\
\text { étudiée }\end{array}$ & $\begin{array}{l}\text { Principes normatifs } \\
\text { Valeurs centrales }\end{array}$ & Objectifs-stratégies & Pratiques concrètes \\
\hline \multirow{6}{*}{ 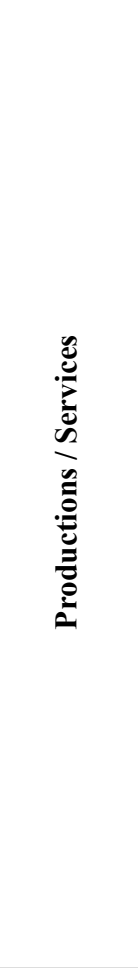 } & \multirow{3}{*}{ Information } & $\begin{array}{c}\text { Journal satirique } \\
\text { Le Couac }\end{array}$ & $\begin{array}{c}\text { Autonomie/indépendance } \\
\text { Autogestion/Empowerment } \\
\text { Anticapitalisme }\end{array}$ & $\begin{array}{c}\text { Ouvrir les consciences par la } \\
\text { pensée critique et l'éducation } \\
\text { Stratégie : politique et } \\
\text { humour }\end{array}$ & $\begin{array}{l}\text { Réseau édition sans structure } \\
\text { matérielle } \\
\text { Fonctionnement coopératif }\end{array}$ \\
\hline & & $\begin{array}{l}\text { Journal régional } \\
\text { Le Mouton Noir }\end{array}$ & $\begin{array}{l}\text { Développement régional } \\
\text { Développement endogène } \\
\text { Culture régionale }\end{array}$ & $\begin{array}{l}\text { Développer identité région. } \\
\text { Émancipation/revitalisation } \\
\text { régionale. Stratégie : } \\
\text { journalisme/pensée critique }\end{array}$ & $\begin{array}{c}\text { Édition journal et formation } \\
\text { stagiaires } \\
\text { Fonctionn. coop. } \\
\text { hiérarchique }\end{array}$ \\
\hline & & $\begin{array}{l}\text { Revue Aube / } \\
\text { Éditions de la } \\
\text { Plume de Feu }\end{array}$ & $\begin{array}{l}\text { Développement durable } \\
\text { Consom. Resp./simpl. Vol. } \\
\text { Développement endogène }\end{array}$ & $\begin{array}{c}\text { Concrétiser dév. durable. } \\
\text { Diffusion pensée écologiste. } \\
\text { Stratégie: } \\
\text { éducation/sensibilisation }\end{array}$ & $\begin{array}{l}\text { Édition revue, organisation } \\
\text { colloques, groupes achats. } \\
\text { Fonctionnement coopératif }\end{array}$ \\
\hline & Communication & $\begin{array}{l}\text { Les Logiciels } \\
\text { Libres }\end{array}$ & $\begin{array}{c}\text { Partage savoir } \\
\text { Empowerment/autogestion } \\
\text { Anticapitalisme }\end{array}$ & $\begin{array}{l}\text { Contourner règles } \\
\text { capitalistes prop. intellect. } \\
\text { Favoriser diff. connaissance } \\
\text { Stratégie : copyleft }\end{array}$ & $\begin{array}{l}\text { Création interactive de } \\
\text { connaissance } \\
\text { Fonctionnement coopératif }\end{array}$ \\
\hline & Tourisme & $\begin{array}{l}\text { Auberge l'Autre } \\
\text { Jardin / Carrefour } \\
\text { Tiers-monde }\end{array}$ & $\begin{array}{l}\text { Économie sociale et } \\
\text { solidaire } \\
\text { Équitabilité échanges écon. } \\
\text { « libéralisme social » }\end{array}$ & $\begin{array}{l}\text { Empowerment tiers-monde. } \\
\text { Offrir service lucratif pour } \\
\text { financer coop. internationale } \\
\text { Stratégie : produit valeur } \\
\text { ajoutée }\end{array}$ & $\begin{array}{l}\text { Petite auberge taylorisée } \\
\text { Fonctionnement } \\
\text { hiérarchique }\end{array}$ \\
\hline & Arts & $\begin{array}{c}\text { Café de la } \\
\text { Petite Gaule }\end{array}$ & $\begin{array}{c}\text { Autogestion/Empowerment } \\
\text { Économie sociale et } \\
\text { solidaire }\end{array}$ & $\begin{array}{l}\text { Ouvrir les consciences par la } \\
\text { pensée critique et } \\
\text { l'éducation. Stratégie : art } \\
\text { et politique. }\end{array}$ & $\begin{array}{l}\text { Vente et sensibilisation. Org. } \\
\text { événements politiques. } \\
\text { Fonctionnement coopératif }\end{array}$ \\
\hline
\end{tabular}




\begin{tabular}{|c|c|c|c|c|c|}
\hline $\begin{array}{l}\text { Champ } \\
\text { d'activité }\end{array}$ & $\begin{array}{l}\text { Secteur } \\
\text { d'activité }\end{array}$ & $\begin{array}{l}\text { Initiative } \\
\text { étudiée }\end{array}$ & $\begin{array}{l}\text { Principes normatifs } \\
\text { Valeurs centrales }\end{array}$ & Objectifs-stratégies & Pratiques concrètes \\
\hline \multirow{7}{*}{ 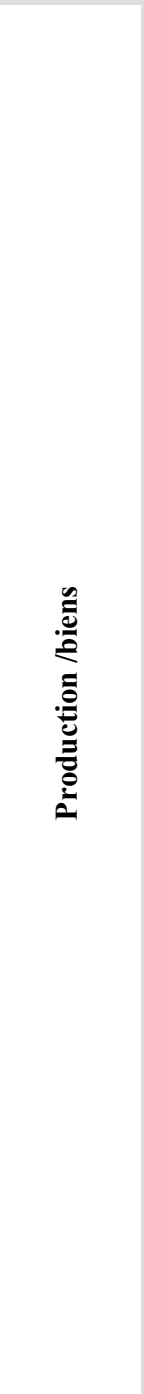 } & \multirow{2}{*}{ Agriculture } & $\begin{array}{l}\text { Agriculture } \\
\text { soutenue par la } \\
\text { communauté }\end{array}$ & $\begin{array}{l}\text { Équitabilité relations éco. } \\
\text { Rapp. directs } \\
\text { consom./produc. } \\
\text { Rapprochement ville/camp. } \\
\text { Développement durable }\end{array}$ & $\begin{array}{l}\text { Contourner règles prod. } \\
\text { capitaliste. Stratégie : offrir } \\
\text { nouveau contrat alimentaire }\end{array}$ & $\begin{array}{l}\text { Renversement schème achat } \\
\text { alimentaire. Coopération } \\
\text { consommateur/producteur } \\
\text { Fonctionnement coopératif }\end{array}$ \\
\hline & & $\begin{array}{l}\text { Fromage Le } \\
\text { Clandestin }\end{array}$ & $\begin{array}{c}\text { Équitabilité relations éco. } \\
\text { Rapp. directs } \\
\text { consom./produc. } \\
\text { Autogestion } \\
\text { Liberté de produire }\end{array}$ & $\begin{array}{l}\text { Contourner règles étatiques } \\
\text { par production aliment } \\
\text { qualité sans contrôle } \\
\text { sanitaire. } \\
\text { Stratégie : clandestinité. }\end{array}$ & $\begin{array}{l}\text { Production-vente clandestine } \\
\text { par petits réseaux. } \\
\text { Fonctionnement collaboratif }\end{array}$ \\
\hline & Immobilier & $\begin{array}{l}\text { Les Écovillages et } \\
\text { fiducies foncières }\end{array}$ & $\begin{array}{c}\text { Développement durable } \\
\text { Autogestion/empowerment } \\
\text { Anticapitalisme } \\
\text { Écologisme }\end{array}$ & $\begin{array}{l}\text { Soustraire sol spéculation. } \\
\text { Communautés durables. } \\
\text { Écologisme concret. } \\
\text { Stratégie : holiste }\end{array}$ & $\begin{array}{l}\text { Création espace exempt } \\
\text { rapports capitalistes. } \\
\text { Autonomie économique du } \\
\text { village. Fonctionnement } \\
\text { coopératif }\end{array}$ \\
\hline & Loisir & $\begin{array}{l}\text { Atelier } \\
\text { communautaire } \\
\text { FreeWheels }\end{array}$ & $\begin{array}{l}\text { Développement durable } \\
\text { Partage compétences } \\
\text { Solidarité communautaire }\end{array}$ & $\begin{array}{l}\text { Consommer moins métal } \\
\text { Favoriser diff. connaissance } \\
\text { Stratégie : coopération } \\
\text { communautaire. }\end{array}$ & $\begin{array}{l}\text { Échange produit (vélo) } \\
\text { /utilisation outils contre } \\
\text { travail sur base forfait fixe. } \\
\text { Fonctionnement coopératif }\end{array}$ \\
\hline & $\begin{array}{c}\text { Biens } \\
\text { industriels }\end{array}$ & SCOPCAT & $\begin{array}{l}\text { Autogestion } \\
\text { Économie sociale } \\
\text { Recyclage }\end{array}$ & $\begin{array}{l}\text { Fabriquer produit à partir } \\
\text { recyclage dans niche } \\
\text { industrielle. } \\
\text { Stratégie : produit valeur } \\
\text { ajoutée }\end{array}$ & $\begin{array}{l}\text { Petite usine taylorisée } \\
\text { Fonctionn. coop. } \\
\text { hiérarchique }\end{array}$ \\
\hline & Mode & $\begin{array}{l}\text { Friperie la } \\
\text { Gaillarde }\end{array}$ & $\begin{array}{c}\text { Économie sociale et } \\
\text { solidaire } \\
\text { Recyclage } \\
\text { Insertion sociale }\end{array}$ & $\begin{array}{c}\text { Créer mode à partir } \\
\text { recyclage. } \\
\text { Insertion jeunes designers. } \\
\text { Stratégie : économie sociale }\end{array}$ & $\begin{array}{l}\text { Vente et sensibilisation } \\
\text { Fonctionn. coop. } \\
\text { hiérarchique }\end{array}$ \\
\hline & $\begin{array}{l}\text { Utilité } \\
\text { publique }\end{array}$ & $\begin{array}{l}\text { Le Santropol } \\
\text { Roulant }\end{array}$ & $\begin{array}{c}\text { Économie sociale et } \\
\text { solidaire } \\
\text { Rapprochement générations } \\
\text { Aliment. qualité/prix } \\
\text { équitable }\end{array}$ & $\begin{array}{l}\text { Rapprocher générations par } \\
\text { service livraison repas bio } \\
\text { bas prix. Stratégie: économie } \\
\text { sociale. }\end{array}$ & $\begin{array}{c}\text { Service aide sociale par le } \\
\text { biais nourriture et livraison. } \\
\text { Fonctionnement } \\
\text { hiérarchique }\end{array}$ \\
\hline \multirow{4}{*}{ 哭 } & \multirow{3}{*}{$\begin{array}{l}\text { Produits } \\
\text { équitables }\end{array}$} & $\begin{array}{l}\text { Café Rico, coop de } \\
\text { torréfaction }\end{array}$ & $\begin{array}{l}\text { Équitabilité relations } \\
\text { économ. } \\
\text { Solidarité internationale } \\
\text { Autogestion/empowerment }\end{array}$ & $\begin{array}{l}\text { Rendre relations } \\
\text { économiques équitables. } \\
\text { Stratégie : coop. internat., } \\
\text { éducation, sensibilisation }\end{array}$ & $\begin{array}{l}\text { Importation, vente, } \\
\text { éducation. Collaboration } \\
\text { avec producteurs } \\
\text { Fonctionnement coopératif }\end{array}$ \\
\hline & & $\begin{array}{l}\text { Boutique } 10000 \\
\text { Villages (Mtl) }\end{array}$ & $\begin{array}{l}\text { Équitabilité relations } \\
\text { économ. } \\
\text { Solidarité internationale } \\
\text { Empowerment tiers-monde }\end{array}$ & $\begin{array}{l}\text { Rendre rel. économiques } \\
\text { équitables. Stratégie : coop. } \\
\text { internat., éducation, } \\
\text { sensibilisation }\end{array}$ & $\begin{array}{l}\text { Importation, vente, } \\
\text { éducation. Collaboration } \\
\text { avec producteurs } \\
\text { Fonctionnement } \\
\text { hiérarchique }\end{array}$ \\
\hline & & $\begin{array}{l}\text { Équita, produits } \\
\text { équitables }\end{array}$ & $\begin{array}{l}\text { Équitabilité relations } \\
\text { économ. } \\
\text { Solidarité internationale } \\
\text { Empowerment tiers-monde }\end{array}$ & $\begin{array}{l}\text { Rendre rel. économiques } \\
\text { équitables. Stratégie: coop. } \\
\text { internat., éducation, } \\
\text { sensibilisation }\end{array}$ & $\begin{array}{l}\text { Importation, vente, } \\
\text { éducation. Collaboration } \\
\text { avec producteurs } \\
\text { Fonctionnement } \\
\text { hiérarchique }\end{array}$ \\
\hline & $\begin{array}{l}\text { Produits } \\
\text { écologiques }\end{array}$ & $\begin{array}{l}\text { Coop La Maison } \\
\text { verte }\end{array}$ & $\begin{array}{l}\text { Développement durable } \\
\text { Économie sociale et } \\
\text { solidaire } \\
\text { Autogestion }\end{array}$ & $\begin{array}{c}\text { Concrétiser dév. durable. } \\
\text { Diffusion pensée écologiste. } \\
\text { Stratégie : } \\
\text { éducation/sensibilisation }\end{array}$ & $\begin{array}{l}\text { Vente de biens à valeur } \\
\text { ajoutée écologique } \\
\text { Fonctionnement coopératif }\end{array}$ \\
\hline
\end{tabular}




\begin{tabular}{|c|c|c|c|c|c|}
\hline $\begin{array}{l}\text { Champ } \\
\text { d'activité }\end{array}$ & $\begin{array}{l}\text { Secteur } \\
\text { d'activité }\end{array}$ & $\begin{array}{l}\text { Initiative } \\
\text { étudiée }\end{array}$ & $\begin{array}{l}\text { Principes normatifs } \\
\text { Valeurs centrales }\end{array}$ & Objectifs-stratégies & Pratiques concrètes \\
\hline \multirow{3}{*}{ 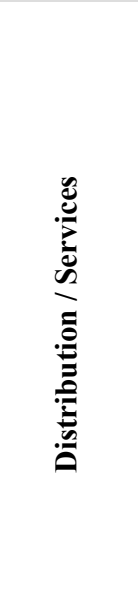 } & \multirow{2}{*}{$\begin{array}{l}\text { Système } \\
\text { d'échanges } \\
\text { locaux }\end{array}$} & $\begin{array}{l}\text { SEL NDG Barter } \\
\text { Network }\end{array}$ & $\begin{array}{l}\text { Partage compétences } \\
\text { Insertion sociale } \\
\text { Solidarité communautaire }\end{array}$ & $\begin{array}{l}\text { Contrer exclusion-solitude- } \\
\text { pauvreté. Stratégie : } \\
\text { coopération communautaire } \\
\text { et reconnaissance } \\
\text { compétence }\end{array}$ & $\begin{array}{l}\text { Échange biens et services sur } \\
\text { base prix unique et égalitaire } \\
\text { Fonctionnement coopératif }\end{array}$ \\
\hline & & SEL Part-SEL & $\begin{array}{l}\text { Partage compétences } \\
\text { Insertion sociale } \\
\text { Solidarité communautaire }\end{array}$ & $\begin{array}{l}\text { Contrer exclusion-solitude- } \\
\text { pauvreté. Stratégie : } \\
\text { coopération communautaire } \\
\text { et reconnaissance } \\
\text { compétence }\end{array}$ & $\begin{array}{l}\text { Échange biens et services sur } \\
\text { base prix unique et égalitaire } \\
\text { Fonctionnement coopératif }\end{array}$ \\
\hline & $\begin{array}{l}\text { Télécommu- } \\
\text { nications }\end{array}$ & $\begin{array}{l}\text { Initiative } \\
\text { Ile-sans-fil }\end{array}$ & $\begin{array}{c}\text { Partage savoir } \\
\text { Empowerment/autogestion }\end{array}$ & $\begin{array}{c}\text { Contourner règles } \\
\text { capitalistes prop. intellect. } \\
\text { Favoriser diff. connaissance. } \\
\text { Stratégie : subversion } \\
\text { techno. }\end{array}$ & $\begin{array}{l}\text { Diffusion gratuite et } \\
\text { interactive d'accès à la } \\
\text { connaissance. } \\
\text { Fonctionnement coopératif }\end{array}$ \\
\hline
\end{tabular}




\section{Publicité du MAMR}

